\title{
Implications on obtained DATA, electronics and DAQ- system by the use of dense detector setups in beta-decay studies.
}

\author{
O. Tengblad, M.J.G. Borge, L.M. Fraile \\ Insto. Estructura de la Materia, CSIC, Serrano 113 bis, E-28006 Madrid, Spain \\ U.C. Bergmann, H.O.U. Fynbo, and K. Riisager \\ Inst for Fysik og Astronomi, Aarhus Universitet, DK-8000 Aarhus C, Denmark \\ B. Jonson, T. Nilsson, G. Nyman, F. Wenander \\ Fysiska Institutionen, Chalmers Tekniska Högskola, S-41296 Göteborg, Sweden \\ for the IS339, IS361 and ISOLDE collaborations CERN
}

\begin{abstract}
Beta decay investigations of light nuclei close to the drip lines have revealed new and intriguing features of nuclear structure. However, to fully investigate the decay pattern of these nuclei new detector systems with an increased granularity have to be implemented. The use of segmented detectors as for example the Double Sided Silicon Strip Detectors has thus proven to give fantastic improvements on the quality of the obtained data. The extra granularity allows for discrimination between sequentially or directly emitted charged particles and thus to follow decay patterns of delayed particle emission.
\end{abstract}

\section{Introduction}

The process of $\beta$-delayed particle emission has been the object of much study during the last few decades. First due to the fact that $\beta$-delayed neutrons play an important role in the control of nuclear reactors and for making decay heat predictions of nuclear fuel. Secondly, as the energy of the particle emitted is directly related to the position of the state fed, important information on the nuclear structure can be obtained. Especially for light nuclei where level density is low it is possible to resolve individual states and perform detailed comparison with theory.

When a quantum mechanical N-body state breaks up into two fragments the system is fully determined by momentum and energy conservation. But this 
is not the case when the final state consists of more than two particles where binary subsystems may influence the break-up.

To fully investigate the decay pattern of the multi-particle break-up of dripline nuclei new detector systems with an increased granularity have to be implemented. However, the introduction of densely packed detectors has also strong implications on the electronics and on the Data Acquisition System to be used. To demonstrate these aspects and to describe our solution to these problems we will use the experimental set-ups and data from two recent ISOLDE experiments; IS339 "The mechanism of $\beta$-delayed two-proton emission." [1], and IS361 "Beta decay asymmetry in mirror nuclei: A=9." [2]

\section{Case Study}

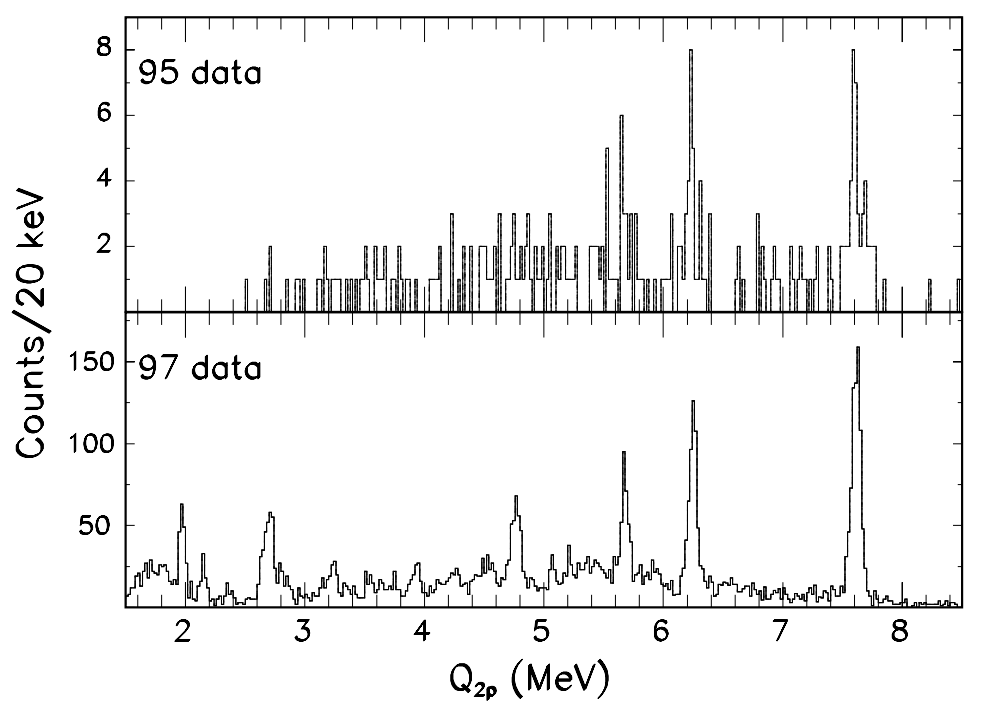

Fig. 1. $\beta$-delayed two proton sum energy $2 \mathrm{p}$ spectra from the decay of ${ }^{31} \mathrm{Ar}$. Upper spectrum from the RUN in 1995 and the bottom spectrum from the RUN in 1997 using a highly segmented detector set-up. The same amount of ${ }^{31} \mathrm{Ar}$ atoms were collected in the two experiments.

Figure 1 shows the $\beta$-delayed two proton spectra from two separate runs from the IS339 experiment using different amounts of detector segmentation. It should be noted that more or less the same yield and total amount of decaying isotopes was collected on foil in the two experiments. The upper spectrum comes from the 1995 RUN where a total of 5 particle detectors were used. The bottom part shows the resulting spectrum from the 1997 RUN, which was explicitly designed to determine the $\beta-2 p$ decay mechanism. In the latter RUN the standard Si- detectors were replaced by a hemisphere (FUTIS [3]) consisting of 15 PIN-diodes covering $2 \pi$ closed from the other side by a Double Sided Silicon Strip Detector (DSSSD) [4] in total yielding a segmentation of 


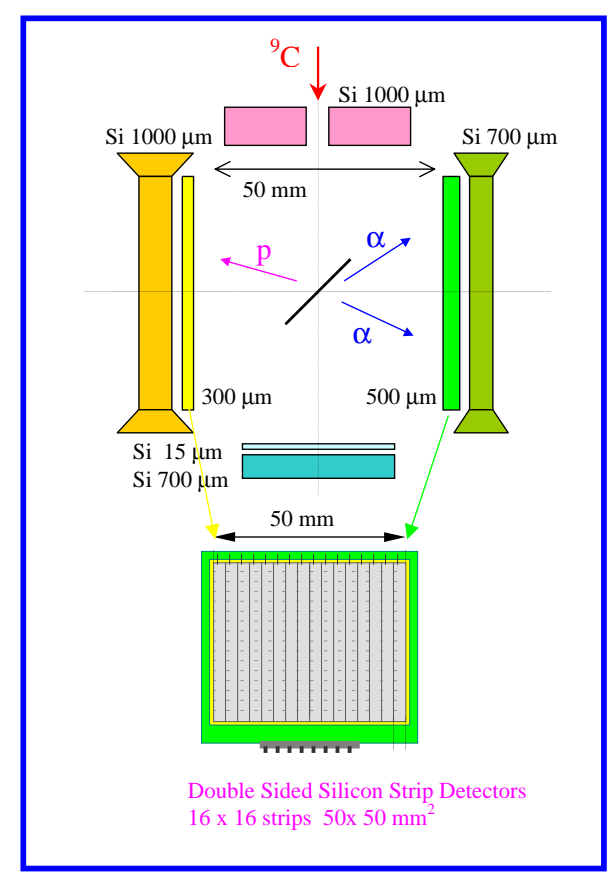

Fig. 2. The experimental set-up used in the IS361 experiment. A highly segmented setup for detecting triple coincidences of the the $\beta$-delayed particle break-up of ${ }^{9} \mathrm{C}$ into $\mathrm{p}+\alpha+\alpha$.

271 detector elements. The improvement in the data with segmentation is clear. The use of the second set-up enabled the decay mechanism to be fully studied for the first time.

\section{The electronics}

The use of highly segmented detectors has strong implications on the electronics to be used. A standard setup consisting of two particle telescopes and a beta monitor (as in the 1995 RUN discussed above) needs some 24 NIM modules. If the two $\Delta \mathrm{E}$ detectors of the telescope are replaced by two DSSSD detectors of $5 \times 5 \mathrm{~mm}^{2}$ with $16 \times 16$ strips as was done in the IS361 experiment (see fig. 2), the 9 parameter experiment has become a 140 parameter experiment needing some 300 NIM modules and 30 crates. For a travelling physicist this becomes impossible. We thus have to search outside our own field of research for new solutions.

Although these kind of detectors have been used for quite some time in reaction experiments it is not trivial to just copy the electronics used there. The dynamic range of interest is different. We study the beta decay at rest so in general the energy of the emitted particles is low: we are therefore hampered by the detector deadlayer and by the fact that little energy is deposited in 
the detectors so that the trigger threshold is in the noise. Compromising we have found two solutions. Firstly, integrated electronics from the Rutherford Appleton Laboratory (RAL). These are modules including 8 shapers and 8 discriminators per motherboard. The dynamic range is $200 \mathrm{MeV}$, but can be changed to $20 \mathrm{MeV}$, which, however, is still about a factor 2-3 too much for us, as a $6 \mathrm{MeV}$ proton is stopped in $300 \mu \mathrm{m}$ Si, which is the detector thickness we use. The main drawback with this module though is the fixed amplification and that the leading edge discriminator setting is common for the 8 channels and so the worst strip determines the trigger threshold. With this system we manage about 500-600 keV trigger threshold, although the energy threshold is as low as $200 \mathrm{keV}$ (this determined from the energy cut of particles in coincidence with the trigger). Secondly, using the CAEN N568B which is a 16 channel NIM Amplifier having both 16 shaped as well as 16 fast outputs. Together with the CAEN C671 CAMAC constant fraction discriminator these two modules can do the same job as two of the RAL units. The advantage with the latter electronics for our case is that here both amplification and threshold can be set independently for each channel, why the actual dynamic range of the system can be matched and the threshold better optimized. With this system the trigger threshold can be lowered to the range of 200-300 keV.

\section{The Data Readout}

To read and store 140 parameters puts severe constraints on the Data Acquisition System. As the beta-decay is an exponentially decreasing random process, the events occur randomly in time, and especially at the drip-line where the halflives are short $(<100 \mathrm{~ms})$, the instantaneous count rate can be very high.

In search of a standalone Data Acquisition System capable of meeting our new needs we found the GSI developed CVC intelligent CAMAC controller and the Multi Branch System (MBS) software [5]. However, due to the special situation of beta decay mentioned above this system has a large total dead time of about $900 \mu \mathrm{s}$. This situation can be improved by using more than one controller (usually an intelligent master controller in VME connected to a CAMAC slave controller via VSB bus).

For the small scaled standalone system we have developed another solution by utilizing the LeCroy FERA system (see fig 3 and 4). Instead of triggering a CAMAC readout for each event the ADCs and TDCs are read via the hardwired FERA bus controlled by a LeCroy 4301 FERA driver into two LeCroy FERA 430216 kword memories working in tandem or ping-pong mode. The overflow output of the memory triggers the CVC to do the CAMAC readout and simultaneously to clear and switch the data storage over to the 


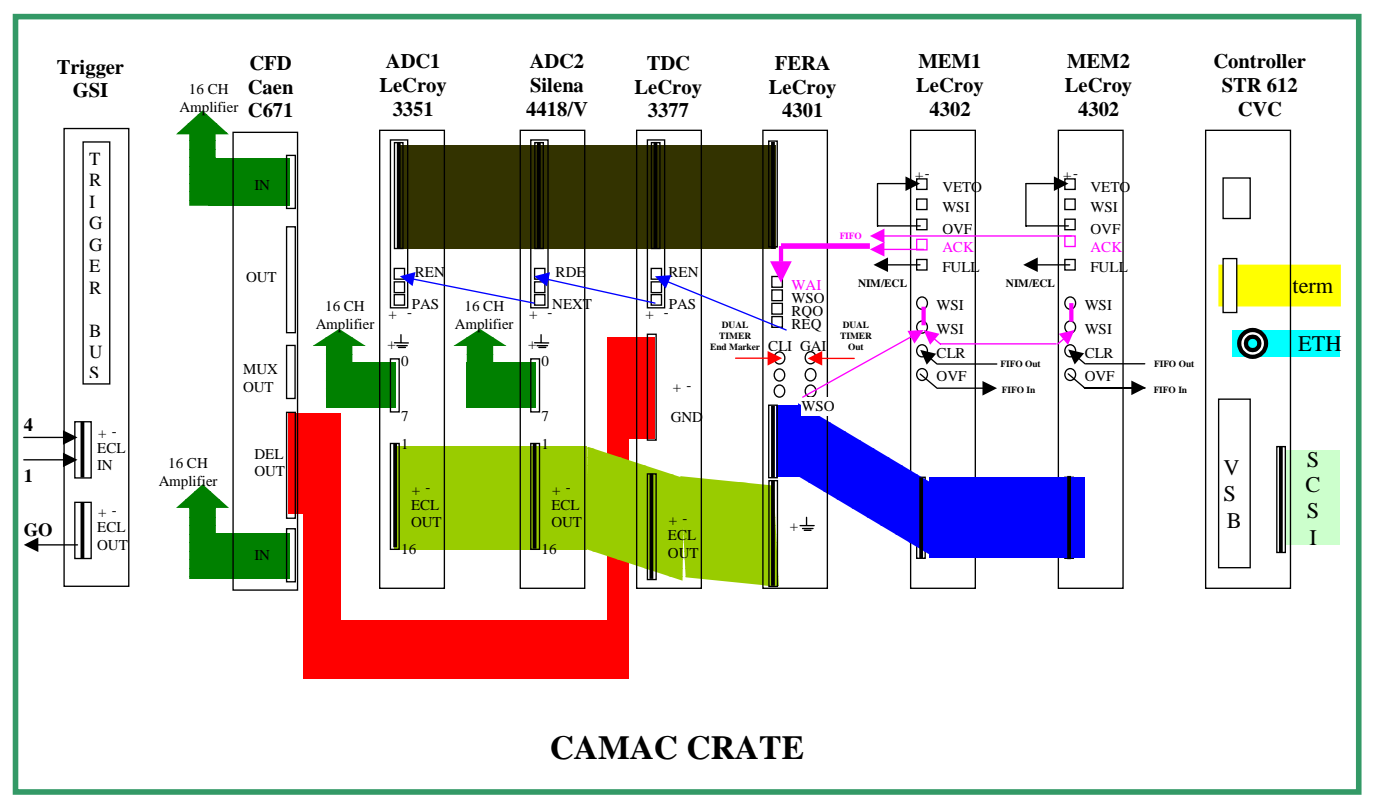

Fig. 3. Schematic diagram of the Standalone Data Acquisition System, utilizing a CVC intelligent CAMAC controller, with the GSI Multi Branch System software together with hardwired FERA readout.

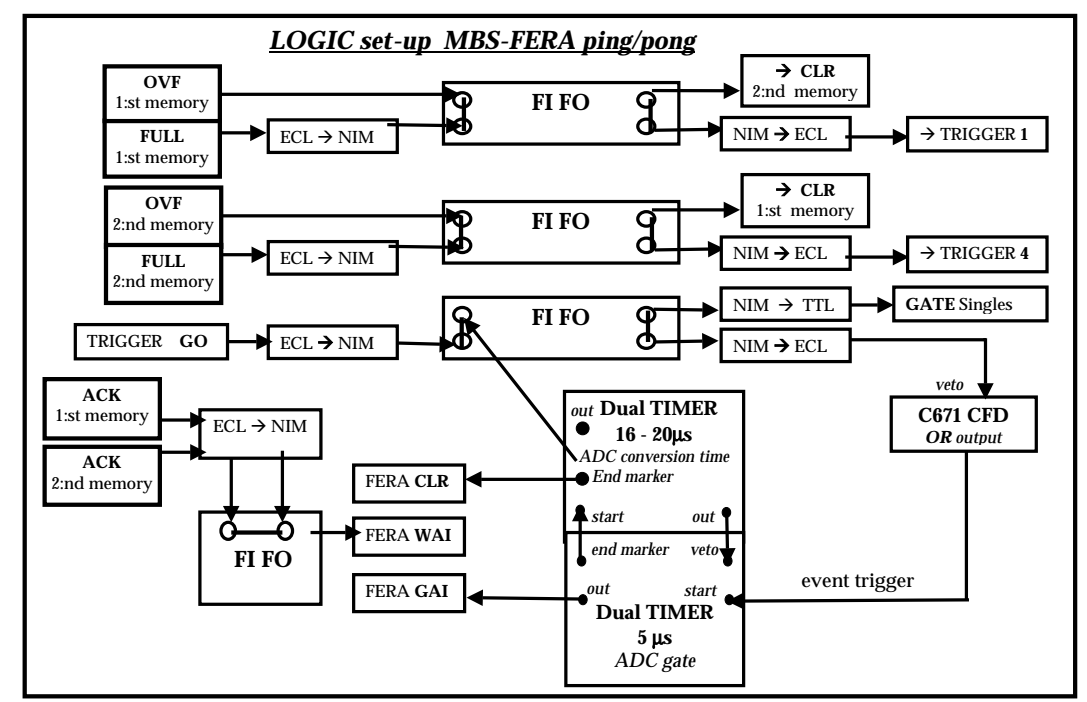

Fig. 4. Schematic diagram of the trigger logic being used in connection with the Standalone Data Acquisition System

second FERA memory. In this way while one memory is being filled the other is read out. The data rate is now only limited by the conversion time of the ADC and by the filling time of the FERA memory. The time it takes to read, mask and store away on tape 16 kwords using the CVC is in the order of 80-100 ms. With an average of 16 words/event (i.e. quadruple coincidence, in the case of the IS361 experiment a $\beta+\mathrm{p}+\alpha+\alpha$ coincidence) an eventrate of 10 $\mathrm{kHz}$ can be obtained free of deadtime. 


\section{Conclusions}

The IS339 and IS361 experiments have successfully shown that by using highly segmented detectors one can study the complex mechanism of multi-particle break- up of drip-line nuclei. We have presented a solution to handle the enormous increase in electronics channels. Especially we have described a standalone Data Acquisition System which can handle an address space of more than 200 parameters at an eventrate of $10 \mathrm{kHz}$ (16 data words/event) free of deadtime.

\section{References}

[1] H.O.U. Fynbo et al., accepted for publication in Nucl. Phys. A

[2] O. Tengblad et al., AIP Proc 495, (Eds. B. Rubio et al, NY,1999)

[3] Dept. of Physics, University of Jyväskylä, FIN-40351 Jyväskylä, Finland.

[4] Micron Semiconductors Ltd., Sussex BN15 8UN, UK.

[5] GSI Multi-Branch System; User Manual and Reference Manual version 3.0, Darmstadt, Germany, 21 September 1999.

[6] FERA - Fast Encoding and Readout ADC; LeCroy Corporation US. 